\title{
Étude d'un système anti-bélier en ligne
}

\section{Study of a water-hammer protection system in line}

F. Massouh

Maître-Assistant

Ecole Nationale Supérieure d'Arts et Métiers

Paris

\author{
R. Comolet
}

\author{
Professeur \\ Université de \\ Pierre et Marie Curie (Paris VI) \\ CNRS - ERA 1028
}

Généralités sur la protection antibélier

Les réseaux hydrauliques sont sujets aux variations de pression en régime transitoire et ces variations peuvent être dans certains cas très importantes. Le choix des canalisations en fonction des pressions transitoires peut augmenter sensiblement le coût du réseau. Pour cela, il est parfois plus intéressant de doter le réseau de moyens de protection antibélier afin de limiter ces pressions transitoires.

Le rôle des appareils de protection consiste à absorber ou compenser en grande partie la variation de débit qui est elle-même à l'origine de régime transitoire; et ceci sous l'effet de faible variation de pression. Ainsi, les variations de débit et de pression ne sont-elles que faiblement ressenties dans le reste du réseau, les variations brusques sont alors transformées en variations graduelles lentes.

Par exemple à la suite d'une fermeture de vanne la surpression est diminuée si le débit coupé est absorbé à l'aide d'une cheminée ou d'un ballon hydropneumatique ou dévié à l'aide d'une soupage de décharge.

De même à la suite d'un arrêt de pompe, la dépression (cavitation éventuellement) est réduite si le déficit du débit est compensé à l'aide de l'un des moyens suivants : ballon hydropneumatique, cheminée, volant d'inertie qui entraîne la pompe pendant un certain temps après l'arrêt du moteur, aspiration auxiliaire (by-pass) et dans certains cas un clapet d'entrée d'air.

Il existe donc plusieurs sortes d'appareils pour se protéger contre les coups de bélier, mais certains ne sont efficaces qu'à la surpression ou à la dépression. L'appareil que nous allons présenter possède l'avantage d'être efficace contre les deux à la fois. De plus, il peut répondre à des conditions d'encombrement qui rendent difficile dans certains cas, l'utilisation des autres moyens.

\section{Présentation de l'amortisseur antibélier en ligne}

Cet amortisseur est simplement constitué d'un tronçon très déformable placé en série avec la conduite à protéger; d'où l'appellation "en ligne ". Cet appareil est donc traversé par la totalité de l'onde et du débit de la conduite contrairement aux autres systèmes antibéliers qui sont installés en dérivation.

L'appareil joue son rôle d'antibélier en réduisant la célérité de propagation sur une partie du parcours de l'onde. La réduction de célérité est obtenue grâce à la grande déformabilité du tronçon constituant l'appareil.

Pour illustrer ce rôle antibélier, nous présentons sur la figure 1 les résultats de plusieurs simulations informatiques fondées sur la méthode des caractéristiques [1, 2]. Le cas simulé est celui de la fermeture instantanée d'une vanne se trouvant à l'extrémité aval d'une conduite alimentée par un réservoir à côte constante à l'amont. La conduite est constituée de deux tronçons $L_{1}=98 \mathrm{~m}$; $D_{1}=0,05 \mathrm{~m} ; L_{2}=2 \mathrm{~m}$ et $D_{2}=0,07 \mathrm{~m}$. Dans le premier tronçon, la célérité est $a_{1}=1200 \mathrm{~m} / \mathrm{s}$, par contre dans le second, plusieurs célérités sont considérées : $a_{2}=200$ puis 100 et $50 \mathrm{~m} / \mathrm{s}$. Le débit coupé est $1 \mathrm{l} / \mathrm{s}$.

La courbe en créneaux est une courbe de référence représentant l'évolution de la pression à l'aval de la conduite après la fermeture de la vanne; ceci pour $L_{1}=100 \mathrm{~m}$ et en l'absence du tronçon $L_{2}$ à basse célérité.

Les autres courbes montrent l'influence de l'introduction du second tronçon $L_{2}$ sur l'évolution de la pression toujours au même point. On constate une nette réduction de la surpression avec la diminution de la célérité $a_{2}$. Par exemple, si $a_{2}=50 \mathrm{~m} / \mathrm{s}$, la surpression de référence sera divisée par quatre environ. Nous reviendrons sur ce sujet au $\S 4$ où nous verrons des résultats expérimentaux plus spectaculaires. 


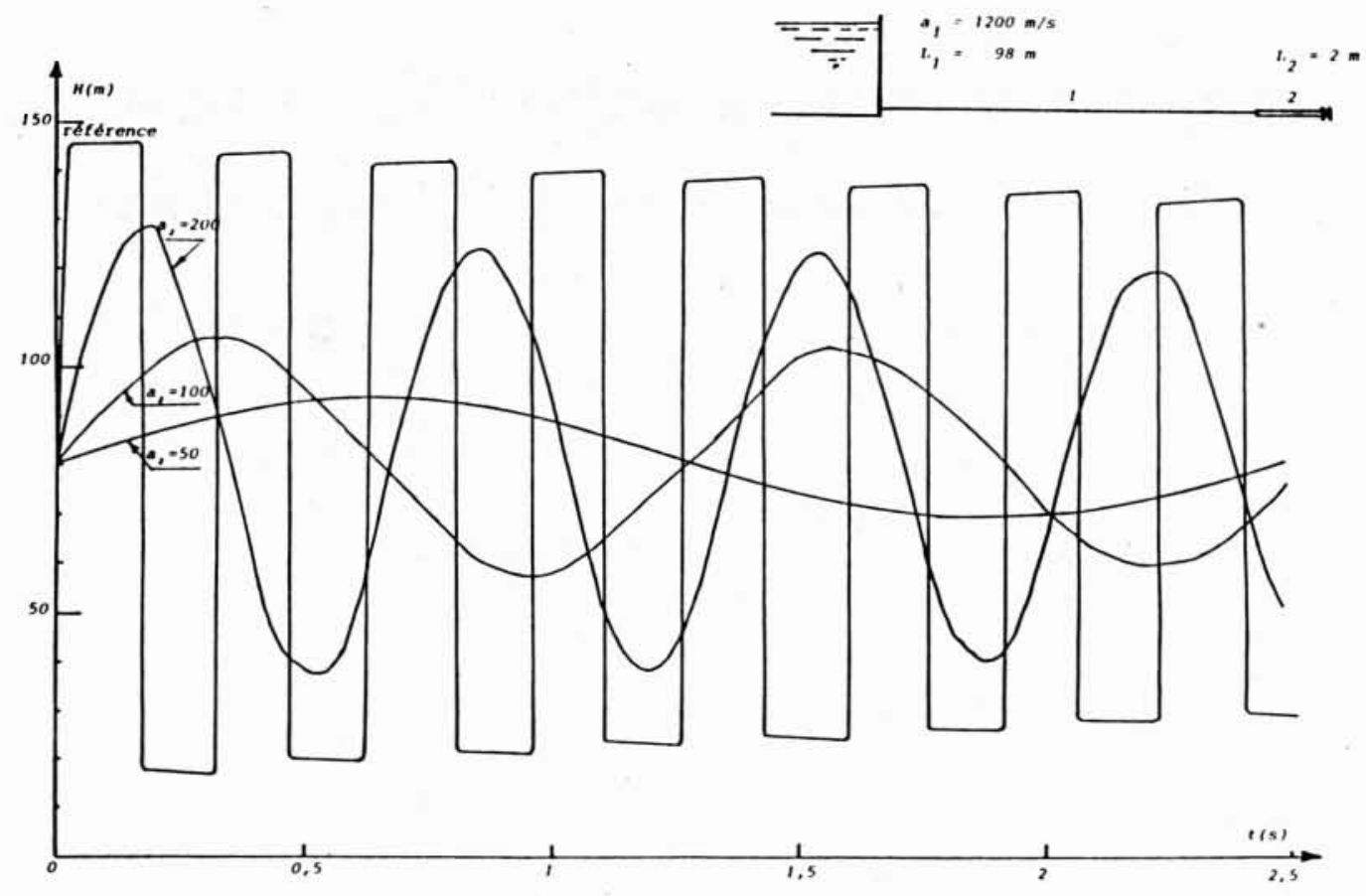

Figure 1 - Influence de la célérité $a_{2}$ sur le coup de bélier.

L'utilisation d'un tronçon déformable comme moyen de protection anti-bélier est une idée développée par plusieurs auteurs mais avec des solutions différentes. Par exemple Réméniéras [3] propose l'utilisation d'une manchette souple contenant de l'air et placée à l'intérieur de la conduite sur une certaine longueur. Pour augmenter davantage la déformabilité du système, Waller [4] propose l'utilisation des balles sphériques en caoutchouc placée dans le liquide, en plus de la manchette en caoutchouc.

Grundy et Fox [5] utilisent, quant à eux, des tubes rectilignes en néoprène gonflés à l'air entourés du liquide et disposés à la périphérie d'un tube central d'écoulement.

L'appareil que nous étudions ici représente une nouvelle solution dont le prototype est schématisé sur la figure 2.

Ici, l'écoulement s'effectue à l'intérieur d'un tube central très déformable constitué d'un manchon en caoutchouc; celui-ci est enfermé dans une enceinte étanche réalisant une chambre d'air périphérique. L'air sous pression dans cette chambre permet de contrebalancer la pression de l'écoulement à l'intérieur du caoutchouc. La haute déformabilité du manchon permet de réduire la célérité de propagation de l'onde à quelques dizaines de mètres par seconde $(10-30 \mathrm{~m} / \mathrm{s})$ dans nos essais.

Dans notre prototype, l'enceinte est simplement réalisée d'un tube en plexiglass pour permettre de visualiser la déformation du manchon en caoutchouc.

Rappelons que dans certaines solutions déjà proposées, il existe un surdimensionnement dû à l'existence de volumes de liquide mort qui ne peuvent être occupés par l'air, ils sont donc inutiles pour la protection antibélier. Par contre, dans la solution que nous proposons tous les volumes sont utilisés et l'occupation de l'espace est optimale.

D'autre part, la haute déformabilité du manchon permet à celui-ci de s'adapter et de se modeler pour assurer à l'écoulement les meilleures conditions de passage.

\section{Étude de la célérité dans l'amortisseur}

La longueur relativement faible de l'amortisseur rend pratiquement impossible l'obtention de la célérité des ondes qui s'y propagent par une mesure de temps. Pour cela la célérité va être déterminée grâce à une méthode dite

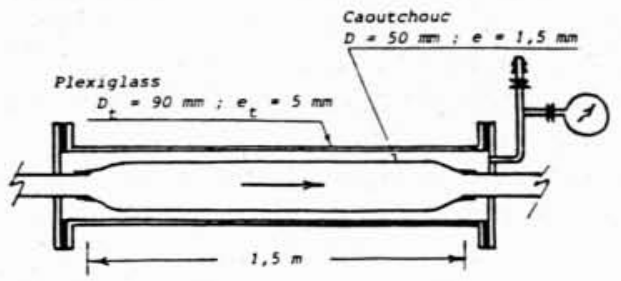

Figure 2 - Présentation schématique du prototype. 
"méthode de gonflage" que nous avons déjà testée dans un travail antérieur [6]. Dans cette méthode la déformabilité $d S / S d P$ de la conduite est mesurée expérimentalement et la célérité est calculée en conséquence à l'aide de l'expression générale de la célérité :

$$
a=\left[\rho\left(\frac{d \rho}{\rho d P}+\frac{d S}{S d P}\right)\right]^{-1 / 2}
$$

où $S$ et $P$ représentent respectivement la section de passage et la pression de l'écoulement, $\rho$ la masse volumique du liquide.

Notre travail [6] a montré qu'en procédant ainsi, on peut obtenir des célérités en bon accord avec celles obtenues directement par la mesure du temps de propagation.

Dans ce qui suit, nous allons donc consacrer notre effort à la détermination de la déformabilité du manchon à l'intérieur de l'amortisseur. Pour cela un modèle théorique de calcul sera élaboré. Ce modèle sera ensuite vérifié expérimentalement.

\section{Calcul de déformabilité du manchon}

La membrane constituant le manchon est soumise simultanément aux actions des forces de pression de l'écoulement à l'intérieur et de l'air dans la chambre extérieure, voir figure 2. Lors d'une variation de pression de l'écoulement, la membrane subit une déformation qui sera déterminée par les lois propres de la membrane et de la chambre d'air. Notre but, ici, est de lier cette déformation à la variation de pression qui la provoque.

Sur la figure 3 nous avons représenté la moitié d'une coupe droite à l'axe de l'amortisseur. Les paramètres sont considérés à un instant quelconque. L'indice 0 sera utilisé pour désigner l'état de référence initial. Nous appelons :

$D$ : diamètre intérieur du manchon

$S$ : surface intérieure du manchon

$e \quad$ : épaisseur du manchon

$S_{a}$ : surface occupée par l'air

$S_{t}$ : surface totale interne de l'amortisseur

$D_{1}$ : diamètre interne de l'amortisseur

$P \quad$ : pression de l'écoulement à l'intérieur du manchon

$P_{a}$ : pression d'air dans la chambre

Le calcul de déformabilité de la membrane sera développé dans le cadre des hypothèses et conditions suivantes :
1) le manchon est amarré aux extrémités et la déformation dans le sens longitudinal sera nulle :

$$
\varepsilon_{1}=0
$$

2) le caoutchouc est incompressible et comme conséquence que la condition précédente, nous pouvons écrire que l'aire du caoutchouc est conservé dans la coupe considérée, d'où :

$$
D \cdot e=D_{0} \cdot e_{0}=\text { cte }
$$

3) le caoutchouc est mince et l'état de contrainte est bidimensionnel.

4) la contrainte circonférentielle dans le manchon est donnée par la loi de Laplace :

$$
\sigma_{t}=\left(P-P_{a}\right) \frac{\mathrm{D}}{2 e}
$$

5) le caoutchouc suit les lois de Hooke qui s'écrivent ici, voir figure (4) :

$$
\begin{aligned}
& \varepsilon_{t}=\frac{\sigma_{t}}{E}-\mu \frac{\sigma_{1}}{E} \\
& \varepsilon_{1}=\frac{\sigma_{1}}{E}-\mu \frac{\sigma_{t}}{E}
\end{aligned}
$$

où

$$
\varepsilon_{\imath}=\frac{D-D_{0}}{D_{0}}
$$

Comme $\varepsilon_{1}=0$ d'après l'équation (2), ceci permet d'écrire :

$$
\frac{D-D_{0}}{D_{0}}=\frac{1-\mu^{2}}{E} \sigma_{t}
$$

et qui devient d'après l'équation (4) :

$$
\frac{D-D_{0}}{D_{0}}=\frac{1-\mu^{2}}{E}\left(P-P_{a}\right) \frac{D}{2 e}
$$

Cette dernière peut être également écrite à l'aide de l'équation (3) comme :

$$
D-D_{0}=\frac{1-\mu^{2}}{2 E e_{0}}\left(P-P_{a}\right) D^{2}
$$

En dérivant cette équation et en introduisant la section $S=\pi D^{2} / 4$, nous obtenons :

$$
\left[\frac{1}{D}-\frac{1-\mu^{2}}{E e_{0}}\left(P-P_{a}\right)\right] \frac{d S}{S}=\frac{1-\mu^{2}}{E e_{0}}\left(d P-d P_{a}\right)
$$

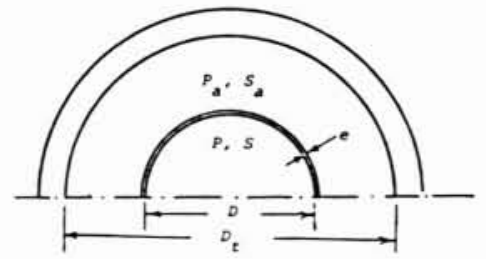

Figure 3 - Section du prototype.

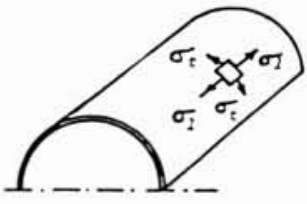

Figure 4 - Contraintes dans la paroi du manchon. 
6) la formation de l'enceinte extérieure est négligeable en comparaison avec la déformation du manchon, nous pouvons donc écrire :

$$
S_{t}=S+\pi D_{0} e_{0}+S_{a}=\text { cte }
$$

Sachant que la surface de caoutchouc reste constante (équation 3), l'équation (8) conduit au résultat :

$$
d S=-d S_{a}
$$

7) l'air est considéré comme un gaz parfait; ce qui permet d'écrire pour l'unité de longueur de l'enceinte :

$$
P_{a} \cdot S_{a}^{\gamma}=P_{a 0} \cdot S_{a 0}^{\gamma}
$$

ou bien :

$$
d P_{a}=-\frac{\gamma \cdot P_{a}}{S_{a}} d S_{a}
$$

où $\gamma=C_{p} / c_{r}$ est le rapport entre les chaleurs spécifiques de l'air à pression constante et à volume constant respectivement.

Enfin pour obtenir la déformabilité $d S / S d P$ nécessaire au calcul de la célérité dans le manchon, nous utilisons les équations (7), (9) et (11) qui donnent :

$$
\frac{\mathrm{dS}}{S d P}=\frac{1}{\frac{E e_{0}}{D\left(1-\mu^{2}\right)}+P_{a}\left(1+\frac{\gamma S}{S}\right)-P}
$$

Dans cette équation les termes $S, S_{a}$ et $P_{a}$ s'expriment tous en fonction du diamètre $D$ qui est à son tour fonction de la pression $P$. Les relations nécessaires pour le calcul sont :

(d'après éq. 8)

$$
\begin{gathered}
S=\pi D^{2} / 4 \\
S_{a}=S_{t}-\pi D_{0} e_{0}-\pi D^{2} / 4
\end{gathered}
$$

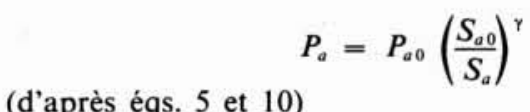

D'autre part, le diamètre $D$ est obtenu en fonction de la pression $P$ à partir des équations $(6),(8)$ et $(10)$ soit :

$$
\begin{aligned}
D-D_{0}= & \frac{1-\mu^{2}}{2 E e_{0}} \\
& {\left[P-\frac{P_{a 0} S_{a 0}^{\gamma}}{\left(S_{t}-\pi / 4 D^{2}-\pi D_{0} e_{0}\right)^{\gamma}}\right] D^{2} }
\end{aligned}
$$

Pour une pression donnée $P$, la solution de la dernière équation donne le diamètre $D$ et permet ensuite de calculer la déformabilité $d S / S d P$, par l'équation (12) et la célérité de propagation par l'équation (1).

Il suffit donc de connaitre les constantes $\mu, E$ et $\gamma$ ainsi que les conditions $P_{\infty 0}, D_{0}$ et $e_{0}$ pour calculer à tout instant le diamètre et la déformabilité du manchon.
Au prochain paragraphe, nous allons effectuer ce calcul et comparer les résultats avec ceux obtenus par la mesure expérimentale pour les mêmes conditions initiales.

\section{Étude expérimentale de la déformabilité de l'amortis- seur}

Nous venons de voir un modèle théorique permettant, à partir de la pression de l'écoulement, de calculer la déformabilité d'un amortisseur donné et d'obtenir ainsi la célérité de propagation. Dans ce qui suit nous exposons la méthode expérimentale utilisée pour vérifier la validité de ce modèle.

Les expériences décrites ci-dessous sont effectuées sur l'amortisseur isolé du reste et fermé aux extrémités, voir figure 5. Une pompe manuelle à piston permet d'injecter à l'intérieur du manchon des volumes connus du liquide. L'amortisseur est équipé de deux manomètres pour mesurer la pression d'eau dans le manchon et la pression d'air dans l'enceinte extérieure.

Comme la longueur $L$ du manchon entre ses amarres est constante et que la compressibilité de l'eau est négligeable par rapport à celle de l'air, tout volume injecté $\Delta V$ se traduit donc par la variation moyenne $\Delta S=\Delta V / L$ de section du manchon.

En l'absence de déformation la contenance du manchon permet de déterminer sa section initiale $S_{0}$. Connaissant le volume initial ainsi que les volumes injectés, on peut alors déterminer à tout moment la section du manchon.

La mesure de la variation de pression consécutive à l'injection $\Delta V$ permet de déterminer une valeur moyenne $\Delta S / S \Delta P$ de la déformabilité. Pour plus de précision et comme la déformabilité est fonction de la pression, nous avons opéré par injections successives pour obtenir toute une relation entre la section et la pression. La courbe $S(P)$ ainsi obtenue et sa tangente $d S / d P$ permettront de connaitre la déformabilité sur tout un domaine de pression.

\section{Comparaison des résultats}

La figure 6 montre l'évolution de la pression d'eau au cours du gonflage et ceci pour trois séries de mesures. Dans ces mesures le volume initial d'eau est toujours le même et correspond au volume du manchon sans déformation. Par contre, la pression initiale d'air varie d'une série de mesures à l'autre : $P \mathrm{a} 0=1$ puis 1,5 et 2 bars en pression absolue.

Pour chaque série de mesures, l'équation (13) est également utilisée pour calculer l'évolution de la pression d'eau dans le manchon. Ce calcul est fait à partir du diamètre du manchon $D$ dont les variations sont connues au cours du gonflage.

Dans le calcul nous avons supposé que l'air subit une transformation isotherme $\gamma=1$; ceci est vérifié expérimentalement comme nous pouvons le constater sur la figure 7 représentant l'évolution de la pression d'air au cours du gonflage. Ce résultat s'explique par ailleurs par les faits suivants :

- la transformation est relativement lente;

- la variation volumique produite à chaque injection est faible; 


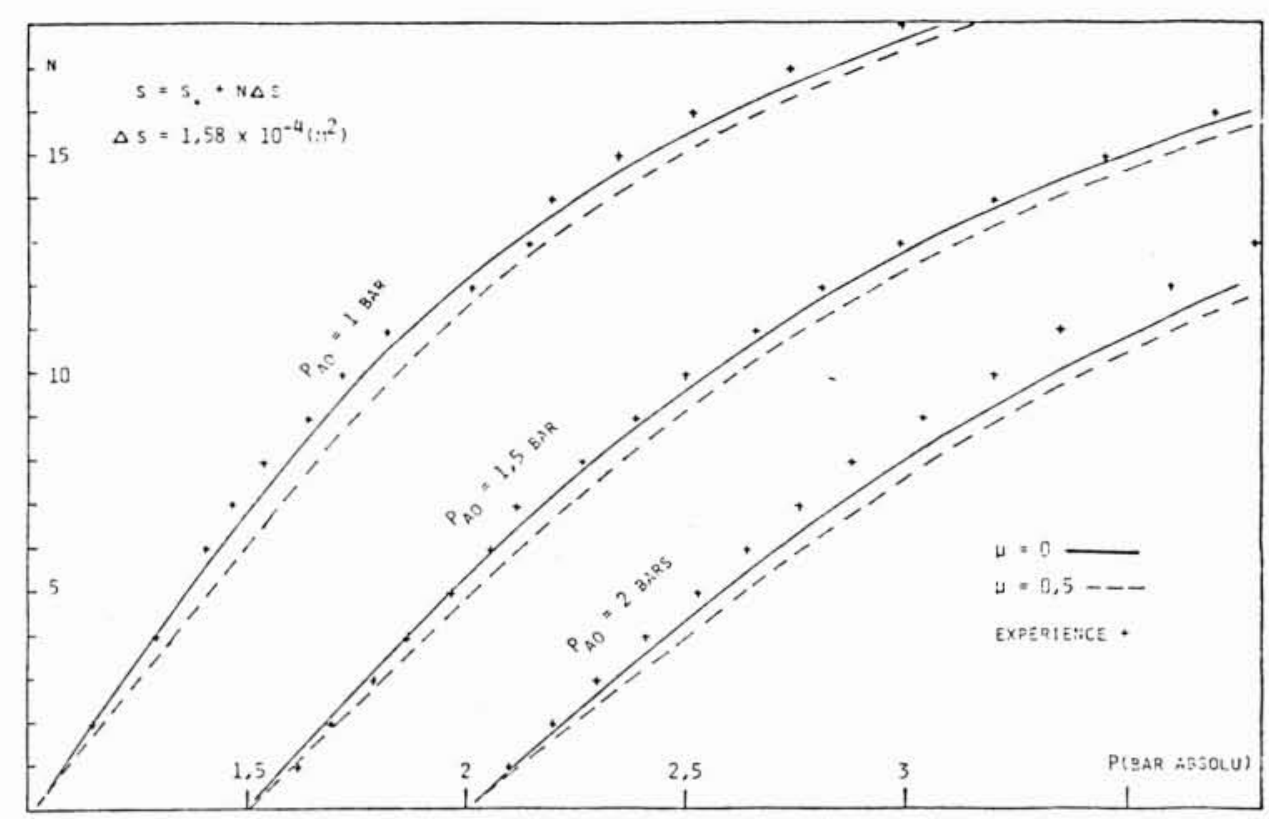

Figure 6 - Relation entre section et pression d'eau dans le manchon.
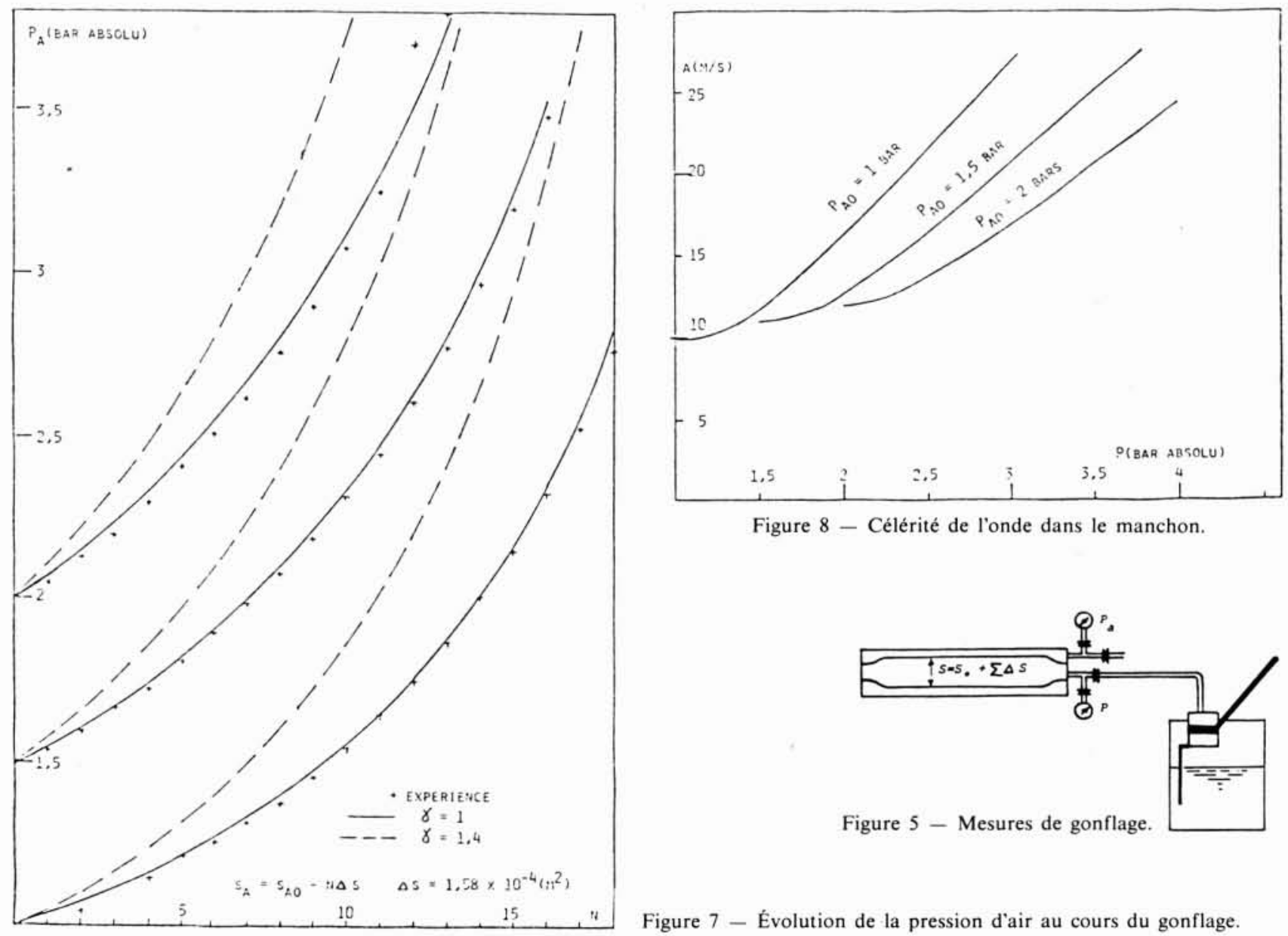

Figure 8 - Célérité de l'onde dans le manchon.

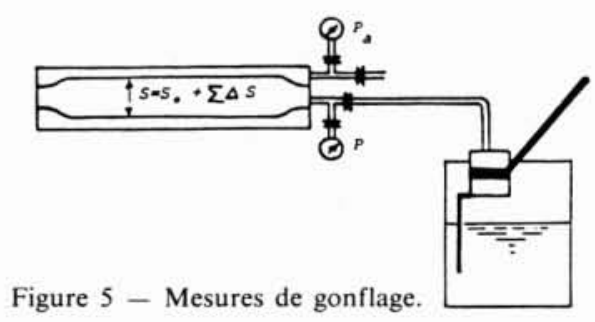

Figure 7 - Évolution de la pression d'air au cours du gonflage. 
- la capacité calorifique de la masse d'eau et des parois extérieures est de loin supérieure à celle de la masse d'air.

Par ailleurs, nous avons effectué le calcul de la déformabilité du manchon en faisant deux hypothèses sur la contrainte longitudinale :

1) calcul avec contrainte longitudinale,

2) calcul sans contrainte longitudinale, $\mu=0$.

On admet que le module de Poisson du caoutchouc est $\mu=0,5$. La valeur $1,9 \times 10^{6}$ Pascals est retenue pour son module d'élasticité; valeur déterminée expérimentalement par un essai de traction sur un échantillon du caoutchouc.

L'examen des résultats figure 6 montre que :

- un bon accord est obtenu entre les calculs et les résultats expérimentaux;

- cet accord est meilleur dans le cas du calcul sans contrainte longitudinale $(\mu=0)$, ce qui montre que le manchon se déforme comme un ensemble d'anneaux circulaires sans aucune influence mutuelle entre eux.

\section{Calcul de la célérité}

Comme nous l'avons déjà expliqué au début du paragraphe 3 nous pouvons maintenant calculer la célérité de propagation de l'onde dans l'amortisseur à l'aide de l'équation (1) et des résultats obtenus par le gonflage. Ce calcul est présenté sur la figure 8 pour les trois séries de mesures. Précisons que dans ce calcul nous avons utilisé l'équation (13) et le cas $\mu=0$ à la place des résultats expérimentaux; l'écart entre les deux étant faible voir figure 6.

En examinant les courbes de la célérité, on constate que celle-ci croît lorsque la pression augmente dans le manchon. D'autre part, la pression initiale de l'air $P_{\infty 0}$ joue un rôle important pour déterminer la loi célérité-pression. $\mathrm{Au}$ début des courbes le manchon n'est pas déformé; la pression transmurale $P-P_{a 0}$ est nulle et la célérité est plus grande là où $P_{a 0}$ est plus grand.

Par contre, pour une pression d'eau $P>P_{\infty 0}$ la célérité augmente plus vite dans le cas où $P_{a 0}$ est plus faible.

Pour l'ensemble des mesures de gonflage, on obtient des célérités de l'ordre de 10 à $28 \mathrm{~m} / \mathrm{s}$. Ces faibles valeurs laissent prévoir une bonne efficacité de l'amortisseur comme moyen de protection anti-bélier.

Signalons enfin que la célérité diminue encore dans un cas de dépression $P<P_{a 0}$. Le manchon devient alors un tube collabable. La valeur minimale de la célérité est atteinte lorsque les parois du manchon viennent en contact [7].

Éssai de l'amortisseur dans un réseau

Comme tout appareil anti-bélier, l'efficacité de l'amortisseur en ligne dépend aussi du réseau où il est installé et de son emplacement dans le réseau. Pour juger de cette efficacité, il faut donc étudier le fonctionnement d'ensemble de l'appareil avec le reste du réseau.
L'étude expérimentale présentée ici, est faite sur le réseau d'étude de coup de bélier du laboratoire d'hydraulique de I'ENSAM. Le schéma de l'installation est présenté sur la figure 9. Une pompe avec une dérivation de fuite à l'amont permettent le choix du point de fonctionnement de l'installation. Ensuite, vient une conduite d'acier d'environ $90 \mathrm{~m}$ de longueur et $25 \mathrm{~mm}$ de diamètre.

Complètement à l'aval, une vanne de manœuvre et un capteur de pression permettent de créer et d'enregistrer le coup de bélier. Juste au voisinage de la vanne le prototype est installé dans une boucle avec un jeu de vannes permettant de faire passer le coup de bélier par l'une ou l'autre des deux branches.

Ainsi, nous avons pu enregistrer les variations de pressions liées au coup de bélier dans la conduite sans ou avec protection. La figure 10 montre les enregistrements obtenus pour un débit coupé $Q=1,121 / \mathrm{s}$. La surpression atteint 26 bars environ en l'absence de protection. L'onde de surpression se propage vers l'amont pour s'y réfléchir et revenir en onde de dépression. Lorsque cette dépression arrive à la vanne fermée, elle sera théoriquement doublée. Mais, en pratique, la pression ne descend pas au-dessous de la pression de vapeur saturée de l'eau et on observe une cavitation sévère surtout dans les premiers créneaux de la figure 10. Le phénomène transitoire dure un certain temps avant d'atteindre le régime permanent final.

Dans le cas où le réseau fonctionne avec l'amortisseur en ligne et pour le même débit coupé, la figure 10 montre que la surpression n'existe pratiquement plus. La pression évolue très lentement vers le régime permanent final et les oscillations ont une période relativement grande.

\section{Conclusion}

Dans cette étude, nous avons présenté un système de protection anti-bélier utilisant les propriétés des tubes hautement distensibles ainsi que la compressibilité de l'air.

Le rôle anti-bélier de ce système est mis en évidence à la fois par le calcul et l'expérience effectuée sur prototype.

Un modèle théorique de calcul de la célérité dans ce système a été proposé et confronté aux résultats donnés par une méthode volumétrique expérimentale. L'accord entre les deux approches s'avère bon dans des conditions que nous avons précisées.

\section{Remerciements}

Les auteurs tiennent à remercier Messieurs Sirot, Raynal et Raze, ingénieurs ENSAM ainsi que tout le laboratoire d'Hydraulique de l'ENSAM pour leur collaboration efficace à ce travail. 


\section{Références bibliographiques}

[1] LISTER M. - Méthodes mathématiques pour calculateurs arithmétiques, Ouvrage Présenté par Ralston A. et Wilf H.S., Dunod 1965, Paris.

[2] MEUNIER M. - Coups de bélier dans les réseaux sous pression, Edition ENGREF 1978 Paris.

[3] RÉMÉNIÉRAS G. - Dispositif simple pour réduire la célérité des ondes élastiques dans les conduites en charge, $\mathrm{La}$ Houille Blanche. Numéro spécial A, pp. 172-196, 1952, France.

[4] WALLER E.J. - Pressure surge control in pipeline systems, Oklahoma State University, Pub. 102, Stillwater, 1958, U.S.
[5] GRUNDY A.K., et FOX J.A. - The effect of compressible pipe-liners on pressure transient generation, B.H.R.A. Pressure Surge, Paper E5, March 1980, Canterbury, England.

[6] MASSOUH F. - Célérité des ondes et coups de bélier dans les conduites élastiques et visco-élastiques, Thèse de Docteur-Ingénieur, Université de Paris VI, 1979.

[7] RIBREAU C. - Écoulements et propagations des ondes dans les conduits collabables, Thèse de $3^{\mathrm{e}}$ cycle, Université de Paris VI, 1978.

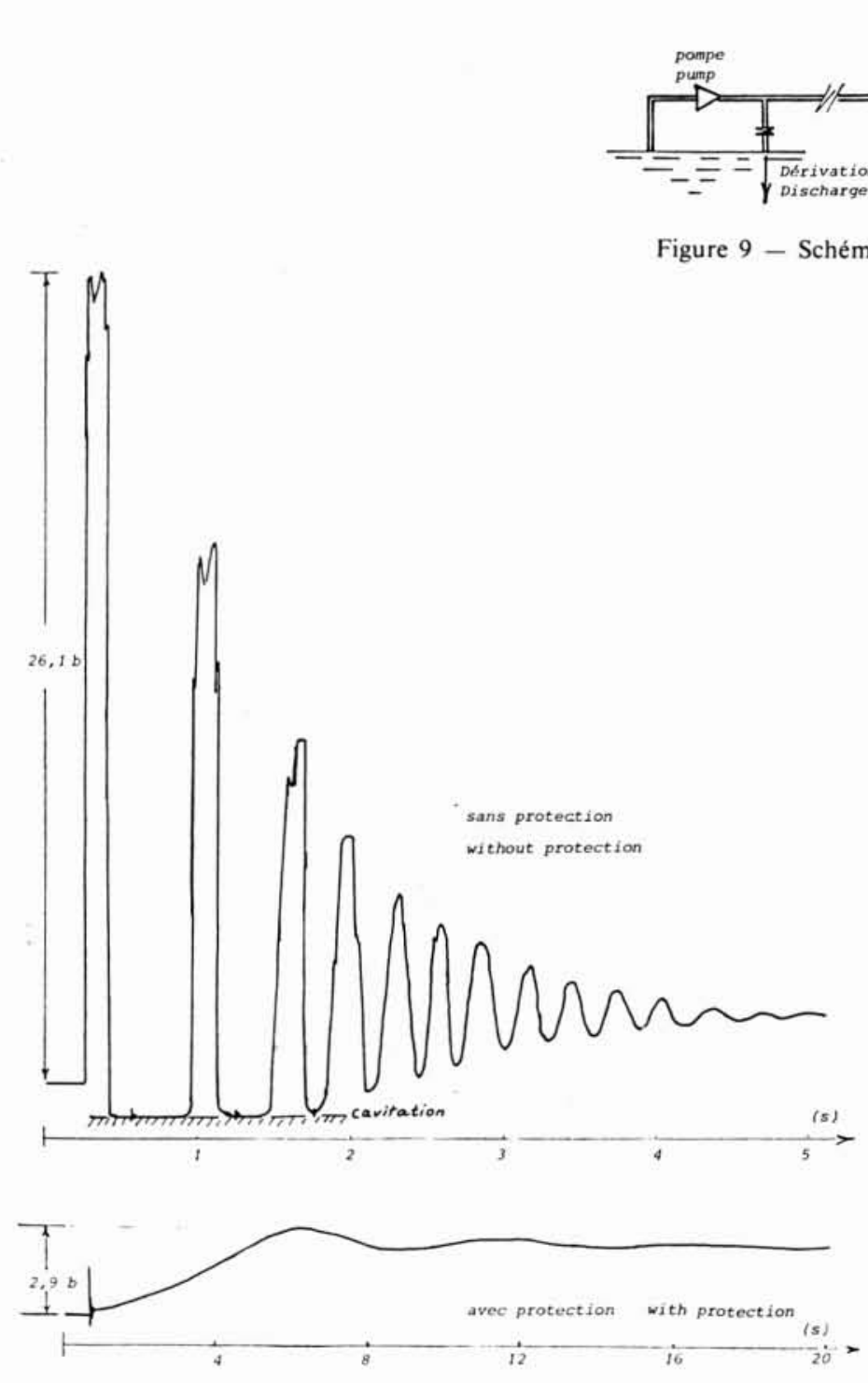

Figure 10 - Comparaison entre les mesures du coup de bélier avec ou sans protection. 


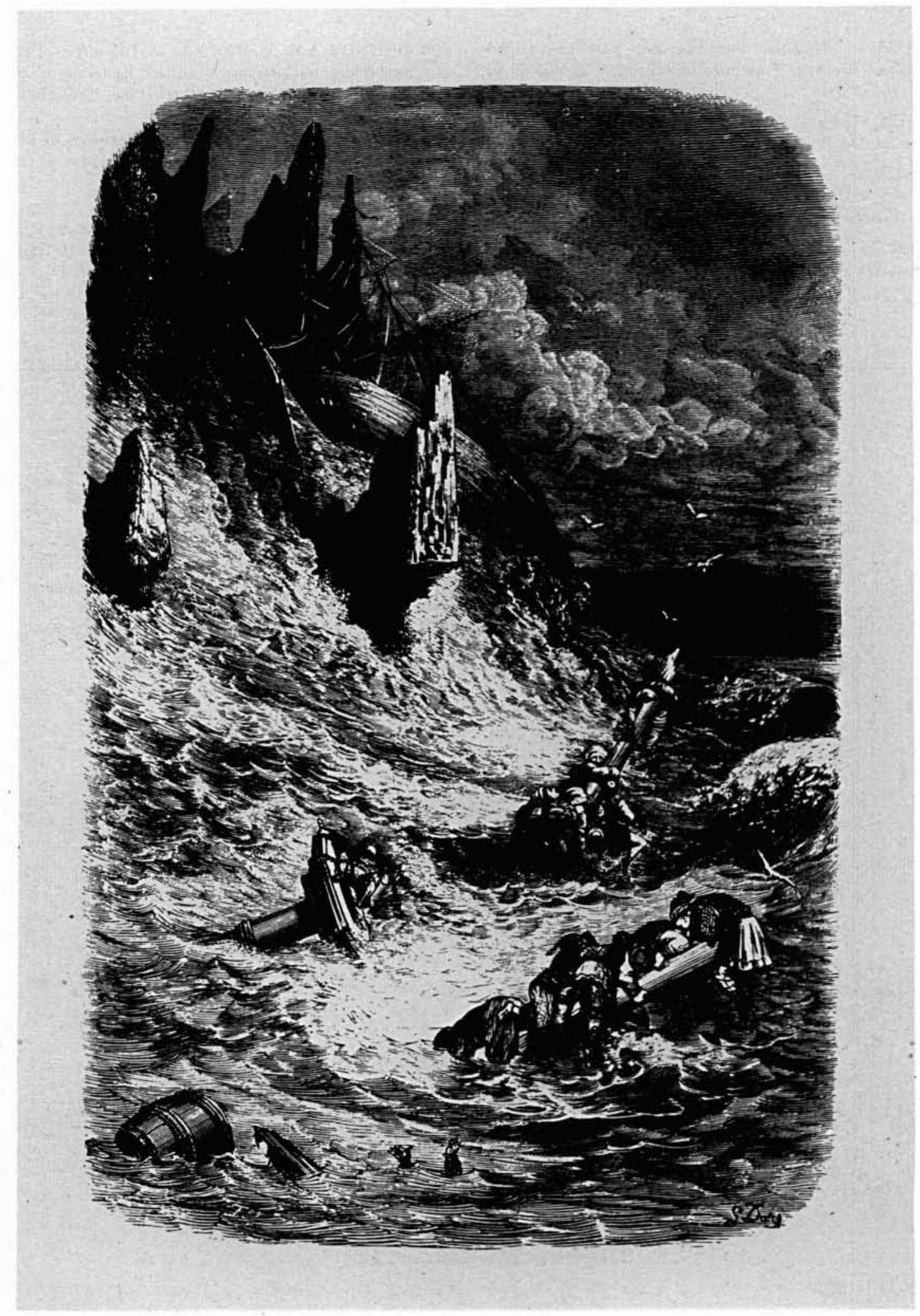

